\title{
Enhanced Band-to-Band Tunneling-Induced-Hot-Electron Injection in P-Channel Flash by SiGe Channel and $\mathrm{HfO}_{2}$ Tunnel Dielectric
}

\author{
Chi-Chao Wang, Kuei-Shu Chang-Liao, Chun-Yuan Lu, and Tien-Ko Wang \\ Department of Engineering and System Science, National Tsing Hua University, \\ Taiwan, R.O.C. \\ Email: 1kschang@ess.nthu.edu.tw
}

\begin{abstract}
SiGe employment is, for the first time, proposed to enhance band-to-band-tunneling-induced hot electron injection (BBHE) in a p-channel flash by the analysis made with two dimensional device simulator MEDICI. Simulation results show that more than 100 times enhancement in the programming speed can be achieved in a proposed p-channel flash with $40 \% \mathrm{Ge}$ in the surface SiGe channel. In addition, a Si-cap layer and $\mathrm{HfO}_{2}$ tunnel dielectric are also incorporated to improve the interface quality. Up to 1000 times enhancement in BBHE injection programming speed is achieved in the case of a p-channel flash memory with surface SiGe layer and $\mathrm{HfO}_{2}$ tunnel dielectric.
\end{abstract}

\section{Introduction}

In order to achieve high injection efficiency, high scalability and low-power operation, a new electron injection scheme with band-to-band tunneling-induced-hot-electron (BBHE) mechanism has been employed in p-channel flash devices [1]. The electron-hole pairs, generated by band-to-band tunneling (BTBT) in the gate-to-drain overlap region, could be accelerated by the lateral electric field toward the channel region and thus obtain enough energy to inject into the floating gate through tunneling oxide, as shown in Fig. 1. A novel structure has been reported to further enhance BBHE injection efficiency [2]. In addition, silicon nitride as tunnel dielectric has been proposed to enhance BBHE programming speed because of its lower dielectric barrier height $(\Delta \mathrm{Ec})$ [3]. In this study, a novel p-channel flash device with a surface strained SiGe layer is demonstrated for the first time by the two-dimensional device simulator MEDICI [4]. A Si-cap layer is also inserted between $\mathrm{SiGe}$ and tunneling oxide to avoid the poor interface between $\mathrm{SiGe}$ and $\mathrm{SiO}_{2}$. Furthermore, $\mathrm{HfO}_{2}$ is introduced as tunneling dielectric, combining with surface $\mathrm{SiGe}$ layer, to gain a better interface [5]. The above three schemes as well as their corresponding band diagrams are illustrated in Fig. 2.

\section{Simulation Method and Device Parameters}

SiGe was employed as the surface channel of p-channel flash device with 10 $\mathrm{nm}$ tunneling oxide and $0.5 \mu \mathrm{m}$ gate length in MEDICI. The amount of $\mathrm{Ge}$ in $\mathrm{SiGe}$ was tuned from $0 \%$ to $80 \%$ as shown in Fig. 2 (a) together with its corresponding energy band diagram. A silicon cap layer, with thickness ranging from $10 \mathrm{~nm}$ to 20 $\mathrm{nm}$, was inserted between tunneling oxide and SiGe as illustrated in Fig. 2 (b). Furthermore, $\mathrm{HfO}_{2}$ is introduced as the tunneling dielectric to gain a better interface 
while $\mathrm{SiGe}$ is served as surface channel, as portrayed in Fig. 2 (c). These three schemes were simulated by identical BBHE injection conditions at drain and gate biases of $-6 \mathrm{~V}$ and $10 \mathrm{~V}$, respectively.

\section{Results and Discussion}

It can be seen in Fig. 3 (a) that as the $\mathrm{Ge}$ in SiGe increases, the programming speed of p-channel flash device with SiGe surface channel enhances from ten to several thousand times. The enhancement in programming speed can be explained by equation (1), which indicates the generation rate of electron hole pair (EHP) from BTBT is inversely exponentially proportional to energy gap:

$G^{B B}=A . B T B T \frac{E^{2}}{E_{g}^{1 / 2}} \cdot \exp \left(-B . B T B T \frac{E_{g}^{3 / 2}}{E}\right)$

The generation rate of BTBT and band-to-band tunneling current $\left(\mathrm{I}_{\mathrm{BB}}\right)$ increase dramatically with increasing Ge content as shown in Fig. 3 (b). Meanwhile, the programming efficiency of -channel flash device with SiGe surface channel remains around $10^{-2}$, indicating this approach can significantly raise the programming speed without sacrificing programming efficiency. With the introduction of a Si-cap, the programming speed decreases with the increasing thickness of silicon cap as shown in Fig. 4 (a). It is found in Fig. 4 (b) that both the injected gate current and band-to-band tunneling current decrease with the increase of thickness of Si-cap layer. The programming efficiency of flash memory cell with SiGe buried layer and $\mathrm{Si}$ cap layer still remains about $10^{-2}$. The contours illustrated in Fig. 5 show that BTBT generation rate of the flash device with Si-cap layer is smaller than that with surface SiGe channel. Results in Fig. 6 show that the drain voltage can be lowered down to $-3.9 \mathrm{~V}$ without altering control gate bias, which means the same programming speed as that of a pure silicon substrate with $\mathrm{Vd}=-6 \mathrm{~V}$ and $\mathrm{Vg}=10 \mathrm{~V}$ can be achieved.

The results shown in Fig. 7 (a) indicate that the employment of either SiGe or $\mathrm{HfO}_{2}$ will improve the programming speed, while the best improvement is achieved when $\mathrm{SiGe}$ and $\mathrm{HfO}_{2}$ are simultaneously applied; in this case, the enhancement can be over 1000 times. Fig. 7 (b) shows that SiGe employment can enhance more BTBT current and $\mathrm{HfO}_{2}$ employment can elevate the injection efficiency; the latter can be attributed to the lower conduction band offset $(\Delta \mathrm{Ec})$ in $\mathrm{HfO}_{2}$. The above simulation results indicate flash devices employing both $\mathrm{HfO}_{2}$ and $\mathrm{SiGe}$ can achieve best BBHE injection.

\section{Conclusion}

A novel p-channel flash device with SiGe band-offset modified channel, together with a Si-cap layer and $\mathrm{HfO}_{2}$ tunnel dielectric to improve interface quality, is proposed to achieve a better BBHE injection. Up to 1000 times enhancement in programming speed can be obtained.

\section{References}

[1] T. Ohnakado, K. Mitsunaga, M. Nunoshita, H. Onoda, K. Sakakibara, N.Tsuji, N. Ajika, M. Hatanaka, and H. Miyoshi, "Novel electron injection method using band-to-band induced hot electron (BBHE) for flash memory with a p-channel cell," IEDM Tech. Dig., 
pp.179-182 1995.

[2] K. Gopalakrishnan, R. Woo, R. Shenoy, Y. Jono, P. B. Griffin, and J. D. Plummer, "Novel very high IE structures based on the directed BBHE mechanism for ultralow-power flash memories," IEEE Electron Device Letters, vol. 26, no. 3, pp. 212- 215, Mar. 2005.

[3] M. She, T.-J. King, C. Hu, W. Zhu, Z. Luo, J.-P. Han, and T.-P. Ma, "JVD silicon nitride as tunnel dielectric in p-channel flash memory," IEEE Electron Device Letters, vol. 23, no. 2, pp. 91-93, Feb. 2002.

[4] Avant! Corp., MEDICI version 2002.4.

[5] Z. Shi, D. Onsongo, K. Onishi, J. C. Lee, and S. K. Banerjee, "Mobility enhancement in surface channel SiGe pMOSFETs with HfO2 gate dielectrics," IEEE Electron Device Letters, vol. 24, no. 1, pp. 34-36, Jan. 2003.

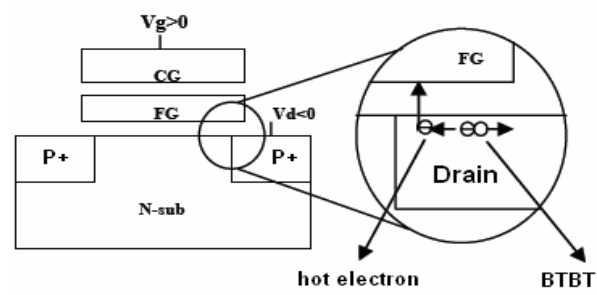

Fig.1. Schematic illustration of the band-to-band- tunneling-induced hot electron (BBHE) injection method in a p-channel Flash memory.
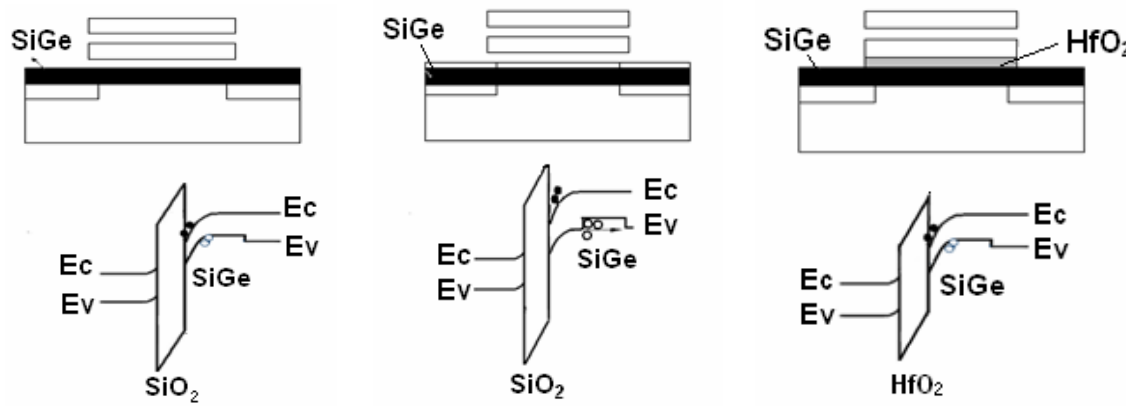

Fig. 2. Schematic illustrations and band diagrams of of a p-channel flash with (a) SiGe surface channel and $\mathrm{SiO}_{2}$ tunneling dielectric (b) SiGe buried layer and $\mathrm{SiO}_{2}$ tunneling dielectric (c) SiGe surface channel and $\mathrm{HfO}_{2}$ tunneling dielectric.

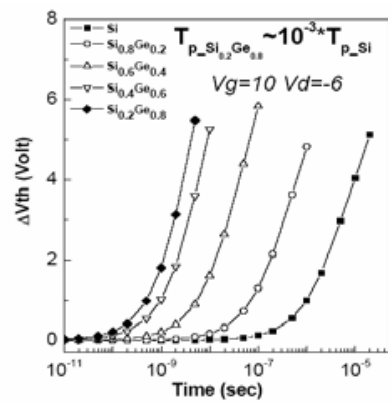

(a)

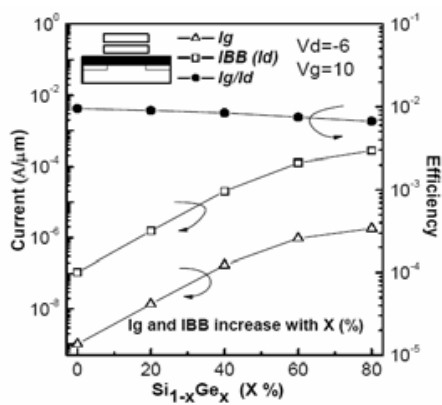

(b)

Fig. 3. (a) Programming speed of a p-channel flash memory with a SiGe surface channel, where the control gate and drain are biased at 10 and $-6 \mathrm{~V}$, respectively. (b) Injected gate current (Ig), BTBT current (IBB), and efficiency for the various Ge contents in a SiGe surface channel. 


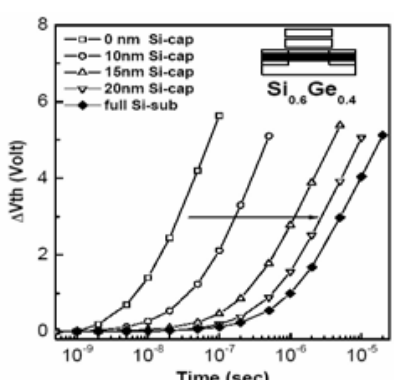

(a)

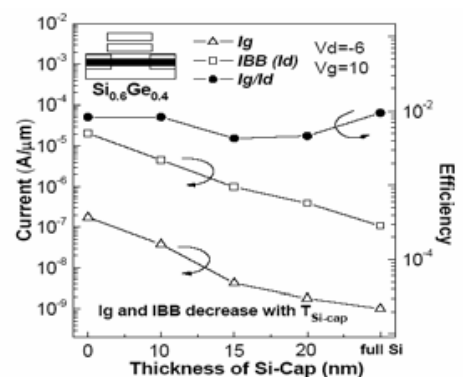

(b)

Fig. 4. (a) Programming speed of a p-channel flash device decreases with increasing the thickness of the Si-cap, where the Ge content in the SiGe epi-layer is fixed at $40 \%$. (b) Injected current (Ig), BTBT current (IBB), and efficiency for the various thicknesses of the silicon-cap.

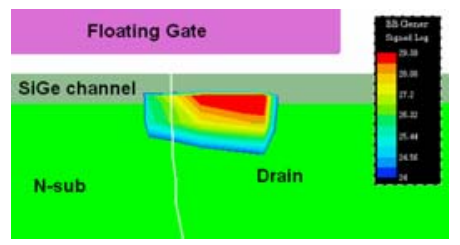

(a)

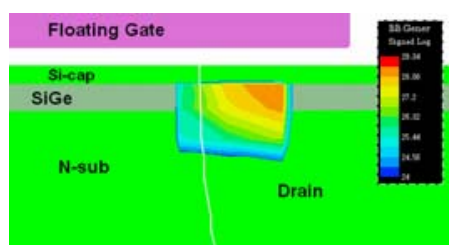

(b)

Fig. 5. 2D generation rate contours of band-to-band tunneling in a p-channel flash (a) with surface SiGe channel (b) with Si-cap and SiGe buried layer.

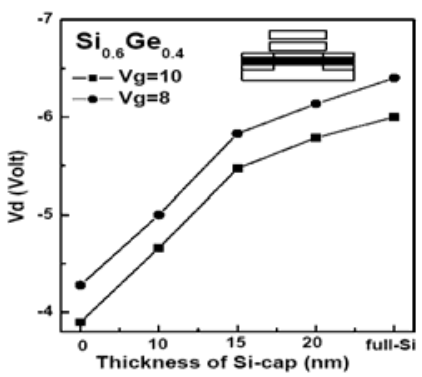

Fig. 6. Drain voltage of programming operation in p-channel flash with $40 \%$ Ge content of $\mathrm{SiGe}$ layer versus various thicknesses of Si-cap.

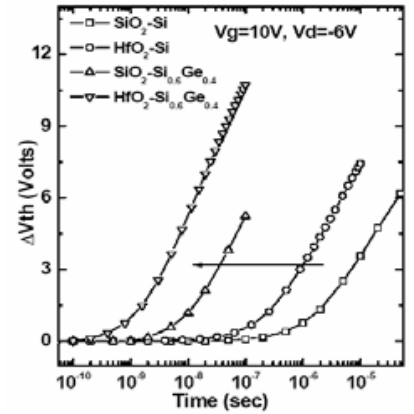

(a)

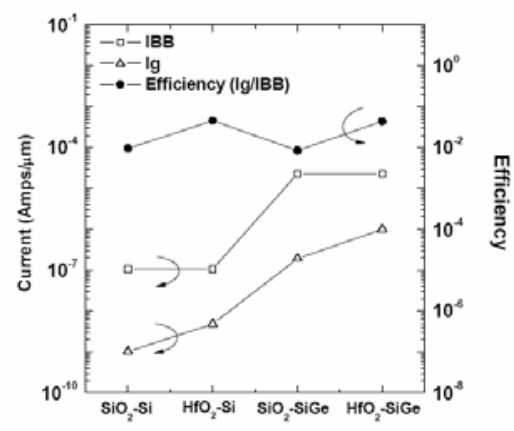

(b)

Fig. 7. (a) The programming speed of p-channel flash device in four kinds of employment, $\mathrm{SiO}_{2} / \mathrm{Si}, \mathrm{HfO}_{2} / \mathrm{Si}, \mathrm{SiO}_{2} / \mathrm{SiGe}$, and $\mathrm{HfO}_{2} / \mathrm{SiGe}$. (b) Injected gate current (Ig), band-to-band tunneling current $\left(\mathrm{I}_{\mathrm{BB}}\right)$, and efficiency for different combination in (a). 